\author{
Matthijs Kox \\ Jan Pompe \\ Astrid Hoedemaekers \\ Peter Pickkers
}

\section{Does subarachnoid haemorrhage affect the innate immune response?}

Accepted: 4 April 2007

Published online: 25 May 2007

(C) Springer-Verlag 2007

An author's reply to this comment is available at: http://

dx.doi.org/10.1007/s00134-007-0655-6.

Sir: Immune suppression following injury to the central nervous system is an important clinical issue because up to $50 \%$ of brain-injury patients develop infection, especially in the first few days following the insult [1]. Recently Naredi et al. [2] tried to establish the immunomodulating effects of the sympathetic nervous system following subarachnoid haemorrhage. The authors found increased norepinephrine spillover following subarachnoid haemorrhage, indicating sympathetic activation, and elevated levels of circulating proinflammatory cytokines and complement such as $\mathrm{C} 3 \mathrm{a}$, interleukin 6 and C5b-9. These parameters were measured to evaluate the relationship between sympathetic nervous system activity and the inflammatory response status, but no quantitative correlation between these two systems was found. Following tissue trauma, as in subarachnoid haemorrhage, circulating levels of pro-inflammatory cytokines may not provide a good measure of the innate immunity because these levels are mainly the result of the trauma itself [3] and do not reflect the innate immune response to a subsequent trigger. Therefore the reported rise and subsequent normalisation in the pro-inflammatory mediators after subarachnoid haemorrhage observed in this study is not surprising.

To evaluate the status of the immune response after subarachnoid haemorrhage or other tissue trauma ex vivo stimulation of immune cells obtained from these patients with lipopolysaccharide or another inflammation-provoking compound would be more appropriate. Moreover, in light of the recently identified "cholinergic anti-inflammatory pathway" future studies into this field should probably consider not only the sympathetic activity but also the activity of the parasympathetic autonomic nervous system (vagal activity) when evaluating the modulation of the immune response [4]. This relatively newly discovered pathway also seems to be relevant in patients with subarachnoid haemorrhage. Apart from an increase in plasma catecholamine concentrations, another study [5] in subarachnoid haemorrhage patients also found a marked increase in parasympathetic activity in the acute phase following subarachnoid haemorrhage. The rise in parasympathetic activity may be the result of the bleeding and brain injury itself or mediated by the subsequent increase in intracranial pressure.

In summary, ex vivo cytokine release to an inflammatory stimulus presents a better measure of the effect of subarachnoid haemorrhage on the innate immunity response than the concentrations of circulating cytokines. Moreover, not only the sympathetic but especially the parasympathetic nerve system may play a pivotal role in the modulation of the innate immune response. Because increased activity of the nervus vagus has been shown to dampen the immune response in both in vitro and animal studies [4], this may represent an interesting new avenue for future research and a promise for new treatment modalities.

\section{References}

1. Helling TS, Evans LL, Fowler DL, Hays LV, Kennedy FR (1988) Infectious complications in patients with severe head injury. J Trauma 28:1575-1577

2. Naredi S, Lambert G, Friberg P, Zall S, Eden E, Rydenhag B, Tylman M, Bengtsson A (2006) Sympathetic activation and inflammatory response in patients with subarachnoid haemorrhage. Intensive Care Med 32:1955-1961

3. Harris BH, Gelfand JA (1995) The immune response to trauma. Semin Pediatr Surg 4:77-82

4. Pavlov VA, Tracey KJ (2005) The cholinergic anti-inflammatory pathway. Brain Behav Immun 19:493-499

5. Kawahara E, Ikeda S, Miyahara Y, Kohno S (2003) Role of autonomic nervous dysfunction in electrocardiographic abnormalities and cardiac injury in patients with acute subarachnoid hemorrhage. Circ J 67:753-756

M. Kox · J. Pompe - A. Hoedemaekers · P. Pickkers ( )

Radboud University Nijmegen Medical Center, Department of Intensive Care

Medicine,

P.O. Box 9101, 6500 HB Nijmegen,

The Netherlands

e-mail: p.pickkers@ic.umcn.nl

Tel.: +31-24-3614170

Fax: +31-24-3541612

\section{J. Pompe}

Radboud University Nijmegen Medical Centre, Department of Surgery, Section Traumatology,

P.O. Box 9101, 6500 HB Nijmegen,

The Netherlands 